\title{
Dose calculation algorithms in external beam photon radiation therapy
}

\author{
Lanchun Lu \\ Department of Radiation Oncology, The James Cancer Hospital, Ohio State University, Columbus, Ohio, USA.
}

Received October 30, 2013; Revised November 12, 2013; Accepted November 13, 2013; Published Online November 22, 2013

\section{Editorial}

The ultimate goal of radiation therapy is to deliver a prescribed dose to a tumor precisely while minimizing dose to the critical structures. Radiation dose is the core of the regime, which also includes dose calculation and delivery of radiation beam. The former is the key component of a treatment planning system. Its accuracy directly impacts the quality of a treatment while its speed heavily affects the clinical flow. This review is focused on photon beam dose calculation algorithms, although some basic concepts can also be applied to other beam modalities.

Why is dose calculation required in radiation therapy? One of the reasons is that we need to plan and simulate the treatment prior to the actual delivery of radiation beam to the tumor. To kill a tumor with radiation, a specific dose needs to be absorbed by the tumor. To make sure this tumor gets the prescribed dose, we need to perform dose calculation by managing radiation beams which are characterized by various parameters in the treatment machine used to deliver the radiation. This process is called treatment planning. In modern radiation therapy, treatment planning is generally performed with computing software by using the patient's images to identify and locate the anatomical structures and the machine parameters to simulate the actual treatment. The result of simulation gives the calculated doses for the target as well as for other regions of interest. The accuracy of dose calculation and the strict quality assurance program is essential in order to make sure that dose delivery to the tumor is $100 \%$ or close to $100 \%$ of the calculated dose.

Radiation dose is defined as the total amount of ionizing radiation energy absorbed by the material or tissues per unit of mass. Hence, dose calculation is computing the energy

Corresponding author: Lanchun $\mathrm{Lu}, \mathrm{PhD}$; The James Cancer Hospital, Department of Radiation Oncology, Ohio State University, $300 \mathrm{~W}$ 10th Avenue, Columbus, OH 43210, USA. Email: lu.281@osu.edu

Cite this article as:

$\mathrm{Lu}$ L. Dose calculation algorithms in external beam photon radiation therapy. Int J Cancer Ther Oncol 2013; 1(2):01025. DOI: $10.14319 /$ ijcto.0102.5 absorbed by the media at any points that radiation beam particles pass or may not pass through, where various physical processes are underway due to the interaction between beam particles and the media. At any point of interest, the dose is contributed by primary beam particles interacting at the point, then scattering from other interacting points in the patient, and the non-primary beam particles leaking from the gantry head. A good dose calculation algorithm is the one that not only can take into account accurately all the physical processes involved in the beam particle-media interaction so that the calculated dose is accurate, but also is fast enough to be used in clinic. Hence, accuracy and speed are the two key factors for a dose calculation algorithm.

The dose calculation algorithm for radiation therapy has been evolving rapidly since the 1950s, mainly attributed to the rapid development in the fields of particle/nuclear physics and computer science which enable us to better understand the physical processes involved in the beam particle-media interaction and to simulate and calculate doses for a complex system within a short time period. Figure 1 illustrates this evolution. From the developing history of dose calculation and the mechanisms used for the dose calculation, we can categorize the dose calculation algorithms into three major groups: (1) correction-based; (2) model-based; and (3) principle-based.

Correction-based algorithm is a type of empirical dose calculation which interpolates or extrapolates dose from some basic measurements in water such as percentage depth dose (PDD) for different field sizes at a certain source surface distance (SSD). The introduction of concepts of tissue-air ratio $(\mathrm{TAR})^{1}$, tissue-phantom ratio (TPR) ${ }^{2}$, and tissue-maximum ratio (TMR) ${ }^{3}$ made this algorithm rather successful in the regime of homogenous media. A typical example of this algorithm is Clarkson's technique ${ }^{4}$ and IRREG ${ }^{5-6}$ that are still commonly used in clinic for manual dose calculation and in some commercial software used for second-hand dose check (RadCalc, Lifeline Software Inc., Austin, Texas, USA). For homogenous media, such as water, this calculation algorithm gives rather accurate results. For a heterogeneous system 
such as a human body with bones and lungs, applying equivalent beam path length can correct part of the heterogeneous effect, but the accuracy is not enough because it cannot take into account the lateral scattering when beam transports in media.

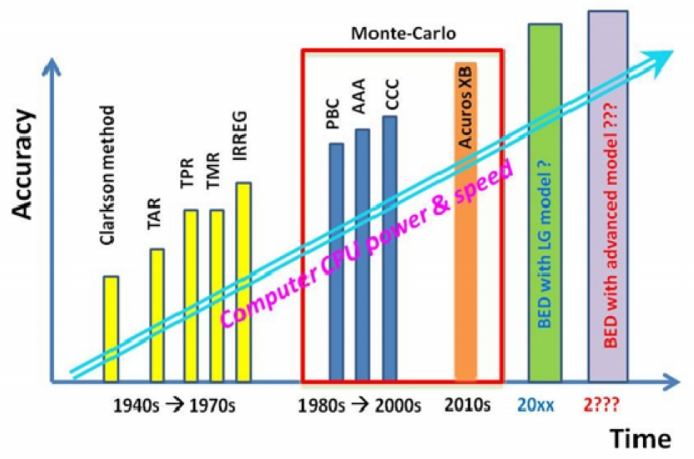

FIG. 1: Evolution of photon dose calculation algorithms.

Model-based algorithm starts first from physics principles and then simplifying the procedure describing the actual physical transport to expedite the calculation. These physical processes have been well studied and are unambiguous: A beam particle interacts with media at a point, releases energy, and then is deposited or scattered away from the primary interaction site. During this process it may create secondary photons and electrons, releasing energy in the scattering path. These physical processes are simplified by using a convolution equation that convolutes the primary photon energy fluence (terma - total energy released in unit of mass) with a kernel that describes the contribution from scattering photons and electrons, and the method is called the convolution algorithm. ${ }^{7-20}$ If the path length is replaced by radiological path length to describe the inhomogeneity of media, the method is called convolution-superposition. The details of how to handle this convolution kernel give rise to sub-difference algorithms that are applied in different commercial treatment plan systems: Pencil Beam Convolution $(\mathrm{PBC})^{12-14}$, the Analytical Anisotropic Algorithm (AAA) 15,18 (Varian Medical System, Inc. Palo Alto, CA, USA ), and Collapse Cone Convolution (CCC) algorithms ${ }^{16,19,20}$ (Pinnacle, $\mathrm{CMS} \mathrm{XiO}$, etc). For homogeneous media such as water, there is not much difference in accuracy for these calculation algorithms. For heterogeneous media, radiological path length is used in place of the actual length to account for the difference in electron density from water, and convolution evolutes to convolution-superposition. The difference in the accuracy of dose calculation on heterogeneous media is determined by how well the kernels of these algorithms can simulate the actual scattering. In PBC, the lateral scattering is considered to be homogeneous, and the inhomogeneity correction only happens in the longitudinal direction which is accounted for by using the equivalent path length con- verted from mass attenuation (hence the electron density of media).

For AAA and CCC, both consider the heterogeneous effect not only in the longitudinal direction, but also in the lateral one. AAA uses Gaussian functions to describe the mean heterogeneous effect in four lateral directions $( \pm \mathrm{X}$ and $\pm \mathrm{y}){ }^{15}, 18$ In CCC, the kernel is replaced by a certain number of discrete elements and the mean for all elements is used to simplify and reduce the calculation time. ${ }^{16,19,20}$ Many research results imply that CCC is more accurate than AAA when an inhomogeneity correction needs to be applied ${ }^{21-23}$, which indicates that CCC handles the lateral scattering better than does AAA in a heterogeneous environment. Monte-Carlo algorithm is not a new technique but has been used as a benchmark to check the accuracy of other dose calculation algorithms. ${ }^{22}$, 24-28 Starting from the first principles of physics, Monte-Carlo simulates the actual physical processes in two major steps which are initiated by a random number seed generation in the target: (1) the radiation beams travel through the accelerator gantry head including the collimator system; (2) collimated beam particles from the gantry head travel through and distribute dose in the patient's body. As Monte-Carlo simulates all the real physical processes in which the beam particles are involved during transportation, the result of its dose calculation should be very accurate.

However, the accuracy is mainly determined by the number of events generated, and this statistical uncertainty is proportional to the inverse square root of the event numbers scored. ${ }^{29}$ For this reason, the speed of Monte-Carlo dose calculation is slow and the process is very time-consuming. The rapid development of computer CPU power greatly enhances the speed of Monte-Carlo dose calculation and makes it possible to be applied in clinic - even though only until very recently the electron Monte-Carlo dose calculation was just implemented into the Varian Eclipse treatment plan system. ${ }^{30}$ The photon Monte-Carlo dose calculation is still very slow and not feasible for use in the clinic. To take advantage of the accuracy of the Monte-Carlo algorithm and avoid its slow speed, simplification of beam particle transport in patient body was made, leading to the AAA and CCC algorithms. ${ }^{15-21}$

Similar to Monte-Carlo algorithm, Acuros XB tries to simulate all the physical processes that beam particles involve instead of generating beam particles one by one in the simulation process, Acuros XB uses a group of Boltzmann transport equations (BTE) to describe all the physical processes involved ${ }^{31}$, and these equations are solved using numerical methods in the computer world, which is much faster than Monte-Carlo (and even the AAA algorithm) but still provides a comparable accuracy to the Monte-Carlo algorithm. The Acuros XB algorithm was recently implemented in the Varian Eclipse Version 10.1, and is available in clinic. ${ }^{32-34}$ 
To test and evaluate the accuracy of a dose calculation algorithm, the best approach is to perform measurements and then compare the measured results with the calculated dose in both homogeneous and heterogeneous media. Many works have been done to evaluate the above-mentioned dose calculation algorithms ${ }^{21-23,30,32-42}$, and the hierarchy of accuracy is as follows: Monte-Carlo algorithm > Acuros $\mathrm{XB}>$ CCC $>$ AAA $>$ PBC $>$ Correction-based methods. ${ }^{21-23,30,32-42}$

With Monte-Carlo and Acuro XB algorithms, you can expect a close to $100 \%$ accuracy of dose calculation if time permits. After more than 60 years of dedicated efforts on the dose calculation algorithm and the development of powerful computer CPUs, the accuracy of dose calculation seems to meet our requirements, and the time needed for calculation in the clinic no longer matters. Are Monte-Carlo and Acuros $\mathrm{XB}$ algorithms the end of the dose calculation game? We should not forget that the ultimate goal of radiation therapy is to kill cancer cells while sparing normal tissues as much as possible. A pure physical dose in non-life media, which does not directly associate with any biological factor in tissues, has no more significance than does a dose calculation algorithm with less accuracy. The next generation of dose calculation algorithms should and is expected to include biological equivalent dose or biological effectiveness dose, which can take into account the actual biological effect for different types of ionization radiation beams and different types of irradiated tissues, and can allow physicians to relate the biological dose more closely to the treatment outcome than what we have today. This is heavily reliant on the progress of radiation biology. Is that going to be a simply biological equivalent dose (BED) with Linear-Quadratic model or BED with a more advanced model?

\section{Conflict of interest}

The authors declare that they have no conflicts of interest. The authors alone are responsible for the content and writing of the paper.

\section{References}

1. Johns HE, Whitmore G, Watson T, et al: A system of dosimetry for rotation therapy with typical rotation distributions. J Can Assn Radiol1953; 4:1.

2. Karzmark CJ, Deubert A, Loevinger R. Tissue-phantom ratios - an aid to treatment planning. Br J Radiol 1965; 38:158.

3. Holt JG, Laughlin JS, Moroney JP. The extension of the concept of tissue-air ratios (TAR) to high-energy x-ray beams. Radiology 1970; 96:437-46.

4. Clarkson JR. A note on depth doses in fields of irregular shape. Br JRadiol 1941; 14:265. 24.
5. Cunningham JR. Scatter-air ratios. Phys Med Biol 1972; 17:42-51.

6. Khan FM, Levitt SH, Moore VC, Jones TK Jr. Computer and approximation methods of calculating depth dose in irregularly shaped fields. Radiology 1973; 106:433-6.

7. Woo MK, Scora DJ, Wong E. The regional Monte Carlo method: a dose calculation method based on accuracy requirement. Med Phys 1998; 25:1866-71.

8. Mackie TR, Bielajew AF, Rogers DW, Battista JJ. Generation of photon energy deposition kernels using the EGS Monte Carlo code. Phys Med Biol 1988; 33:1-20.

9. Ahnesjö A, Aspradakis MM. Dose calculations for external photon beams in radiotherapy. (Topical Review), Phys Med Biol 1999; 44:R99-155.

10. Nilsson M and Knoos T. Application of the Fano theorem in inhomogeneous media using a convolution algorithm. Phys Med Biol 1992; 37: 69-83.

11. Ahnesjo A. Collapsed cone convolution of radiant energy for photon dose calculation in heterogeneous media. Med Phys 1989; 16: 577-92.

12. Mohan R, Chui C, Lidofsky L. Differential pencil beam dose computation model for photons. Med Phys 1986; 13:64-73.

13. Ahnesjö A, Saxner M, Trepp A. A pencil beam model for photon dose calculation. Med Phys 1992; 19:263-73.

14. Bourland JD, Chaney EL. A finite-size pencil beam model for photon dose calculations in three dimensions. Med Phys 1992; 19:1401-12.

15. Sievinen J, Ulmer W, Kaissl W. AAA photon dose calculation model in Eclipse. Varian Medical Systems 2005; RAD \#7170B.

16. McNutt T. The ADAC Pinnacle Collapsed Cone Convolution Superposition Dose Model. ADAC Radiation Therapy Products. www.adaclabs.com

17. Sharpe MB, Battista JJ. Dose calculations using convolution and superposition principles: the orientation of dose spread kernels in divergent $\mathrm{x}$-ray beams. Med Phys 1993; 20:1685-94.

18. Ulmer W, Harder D. A Triple Gaussian Pencil Beam Model for Photon Beam Treatment Planning, Z. Med Phys 1995; 5:25-30.

19. Mackie TR, Bielajew AF, Rogers DW, Battista JJ. Generation of photon energy deposition kernels using the EGS Monte Carlo code. Phys Med Biol 1988; 33:1-20.

20. MackieTR, Reckwerdt PJ, Holmes TW, Kubsad SS, Review of convolution/superposition methods for photon beam dose computation. Proceedings of the Xth ICCR 1990; 20-23.

21. Hasenbalg F, Neuenschwander H, Mini R, Born EJ. Collapsed cone convolution and analytical anisotropic algorithm dose calculations compared to 
VMC++ Monte Carlo simulations in clinical cases. Phys Med Biol 2007; 52:3679-91.

22. Aarup LR, Nahum AE, Zacharatou C, Juhler-Nøttrup T, Knöös T, Nyström H, Specht L, Wieslander E, Korreman SS. The effect of different lung densities on the accuracy of various radiotherapy dose calculation methods: implications for tumour coverage. Radiother Oncd 2009; 91:405-14.

23. Gray A, Oliver LD, Johnston PN. The accuracy of the pencil beam convolution and anisotropic analytical algorithms in predicting the dose effects due to attenuation from immobilization devices and large air gaps. Med Phys 2009; 36:3181-91.

24. Ma CM, Li JS, Pawlicki T, Jiang SB, Deng J, Lee MC, Koumrian T, Luxton M, Brain S. A Monte Carlo dose calculation tool for radiotherapy treatment planning. Phys Med Biol 2002; 47:1671-89.

25. Webb S, Parker RP. A Monte Carlo study of the interaction of external beam X-radiation with inhomogeneous media. Phys Med Biol 1978; 23:1043-59.

26. Kawrakow I. VMC++, Electron and Photon Monte Carlo Calculations Optimized for Radiation Treatment Planning in Advanced Monte Carlo for Radiation Physics, Particle Transport Simulation and Applications: Proc. Monte Carlo 2000 Meeting Lisbon, A. Kling, F. Barao, M. Nakagawa, L. Tavora, and P. Vaz (eds.), Berlin: Springer 2001; 229-236.

27. Reynaert N, Van der Marck SC, Schaart DR, et al. Monte Carlo treatment planning for photo and electron beams. Radiation Physics and Chemistry 2007; 76:643-686.

28. Tertel J, Wulff J, Karle H, Zink K. Verification of a commercial implementation of the Macro-Monte-Carlo electron dose calculation algorithm using the virtual accelerator approach. $Z$ Med Phys 2010; 20:51-60.

29. Jeraj R, Keall P. The effect of statistical uncertainty on inverse treatment planning based on Monte Carlo dose calculation. Phys Med Biol 2000; 45:3601-13.

30. Hu YA, Song H, Chen Z, Zhou S, Yin FF. Evaluation of an electron Monte Carlo dose calculation algorithm for electron beam. J Appl Clin Med Phys 2008; 9:2720.

31. Vassiliev ON, Wareing TA, McGhee J, Failla G, Salehpour MR, Mourtada F. Validation of a new grid-based Boltzmann equation solver for dose calculation in radiotherapy with photon beams. Phys Med Biol 2010; 55:581-98.

32. Kroon PS, Hol S, Essers M. Dosimetric accuracy and clinical quality of Acuros XB and AAA dose calculation algorithm for ste-reotactic and conventional lung volumetric modulated arc therapy plans. Radiat Oncol 2013; 8:149.
33. Fogliata A, Nicolini G, Clivio A, Vanetti E, Cozzi L. Critical appraisal of Acuros XB and Anisotropic Analytic Algorithm dose calculation in advanced non-small-cell lung cancer treatments. Int J Radiat Oncol Biol Phys 2012; 83:1587-95.

34. Han T, Mikell JK, Salehpour M, Mourtada F. Dosimetric comparison of Acuros XB deterministic radiation transport method with Monte Carlo and model-based convolution methods in heterogeneous media. Med Phys 2011; 38:2651-2664.

35. Mesbahi A, Thwaites DI, Reilly AJ. Experimental and Monte Carlo evaluation of Eclipse treatment planning system for lung dose calculations. Rep Pract Onc Radiother 2006; 11:123-133.

36. Van Esch A, Tillikainen L, Pyykkonen J, Tenhunen M, Helminen H, Siljamäki S, et al. Testing of the analytical anisotropic algorithm for photon dose calculation. Med Phys 2006; 33:4130-48.

37. Nishio T, Kunieda E, Shirato H, Ishikura S, Onishi $\mathrm{H}$, Tateoka $\mathrm{K}$, et al. Dosimetric verification in participating institutions in a stereotactic body radiotherapy trial for stage I non-small cell lung cancer: Japan clinical oncology group trial (JCOG0403). Phys Med Biol 2006; 51:5409-17.

38. Knöös T, Wieslander E, Cozzi L, Brink C, Fogliata A, Albers D, Nyström H, Lassen S. Comparison of dose calculation algorithms for treatment planning in external photon beam therapy for clinical situations. Phys Med Biol 2006; 51:5785-807.

39. Lu LC, Yembi-Goma G, Wang JZ, et al. A practical method to evaluate and verify dose calculation algorithms in the treatment planning system of radiation therapy. Int J Med Phys Clin Eng Radiat Oncl 2013; 2:76-87.

40. Rana S, Rogers K. Dosimetric evaluation of Acuros $\mathrm{XB}$ dose calculation algorithm with measurements in predicting doses beyond different air gap thickness for smaller and larger field sizes. J Med Phys 2013; 38: 9-14.

41. Kathirvel M, Subramanian S, Clivio A, et al. Critical appraisal of the accuracy of Acuros-XB and Anisotropic Analytical Algorithm compared to measurement and calculations with the compass system in the delivery of RapidArc clinical plans. Radiat Oncol 2013; 8:140.

42. Rana S, Rogers K, Lee T, Reed D, Biggs C. Verification and Dosimetric Impact of Acuros XB Algorithm for Stereotactic Body Radiation Therapy (SBRT) and RapidArc Planning for Non-Small-Cell Lung Cancer (NSCLC) Patients. Int J Med Phys Clin Eng Radiat Oncl 2013; 2:6-14.

(Dr. Lanchun Lu is an editorial board member of International Journal of Cancer Therapy and Oncology) 